\title{
A review of oral food challenges in children presenting to a single tertiary centre with perceived or true food allergies
}

Meera Thalayasingam ${ }^{1}$, MRCPCH, FRACP, Evelyn Xiu Ling LoO ${ }^{2,3}$, PhD, Michelle Meiling Tan ${ }^{4}$, BSc, MSc, Hugo Van Bever ${ }^{2,4}$, MMed, PhD, Lynette Pei-Chi Shek ${ }^{2,4}$, MBBS, MRCP

\begin{abstract}
INTRODUCTION The prevalence of perceived food allergies exceeds that of true food allergies. Unnecessary food avoidance may increase parental and patient anxiety, reduce quality of life and increase the risk of nutritional deficiency. An oral food challenge (OFC) can provide an objective measure regarding the presence or absence of food allergies in a child. This study reviews the indications for and outcomes of OFCs performed on children.

METHODS A retrospective review was performed on all children who underwent OFCs at the Allergy Unit of the National University Hospital, Singapore, over a three-year period.

RESULTS A total of 197 OFCs were performed among 58 patients (34 male, 24 female). Most of the tests were for allergies to tree nuts $(n=107)$. Among the OFCs, $43.1 \%$ were for foods that were avoided and never eaten due to perceived food allergies, $25.9 \%$ were for foods that had previously resulted in positive skin prick tests (SPTs) and/or immunoassay results, $16.2 \%$ were for foods thought to worsen eczema and $14.7 \%$ were for foods thought to have caused a previous reaction. Of all the OFCs, $5 \%$ were positive, although adverse reactions were mostly cutaneous. Challenge-positive patients had either positive SPTs (wheal $>3 \mathrm{~mm}$ ) or raised serum immunoglobulin E levels to specific foods that they reacted to during the challenges. No episodes of anaphylaxis were reported after the challenge. Most of the patients were able to safely introduce the avoided foods into their diets.

CONCLUSION OFCs provide an objective assessment for suspected food allergies.
\end{abstract}

Keywords: food allergy, indications, oral food challenges, outcomes, safety

\section{INTRODUCTION}

The prevalence of perceived food allergies exceeds that of true food allergies, especially if the estimates are based on selfreported surveys. ${ }^{(1)}$ A substantial number of parents perceive food allergies in their children based on subjective reactions or allergy tests alone. ${ }^{(2)}$ Others readily draw a connection between certain foods and chronic illnesses such as eczema. ${ }^{(2)}$ In addition, cross-reactive foods are avoided in children with certain food allergies, as parents fear that their children will react even if they have not been tested for or eaten these foods. This is seen in many peanut-allergic children who avoid tree nuts but have not been tested for clinical reactivity to tree nuts. Similarly, demonstrating sensitisation alone, via positive skin prick tests (SPTs) or raised serum immunoglobulin E (IgE) levels, does not necessarily equate to having an allergy; ${ }^{(3)}$ and eczema flare-ups are also not always related to foods. ${ }^{(4)}$ Thus, it is not uncommon for children seen at allergy clinics to be on strict elimination diets based on incomplete information regarding potential food allergies. While the vast majority of these children are not allergic to the omitted foods, they are made to adhere to diets that are similar to those of patients with life-threatening reactions to these foods; this results in unnecessary food avoidance, an impaired quality of life, potentially poor weight gain and malnutrition. ${ }^{(5)}$
An oral food challenge (OFC) can be used to provide parents with an objective measure of their child's true food allergies. As no local data is available on the use of OFCs to measure the presence or absence of food allergies, a retrospective chart review was carried out to describe the indications that prompted OFCs and the outcomes of the OFCs conducted.

\section{METHODS}

This study was a retrospective review conducted on patients aged $\leq 18$ years who underwent OFCs at the Paediatric Allergy Clinic of the National University Hospital, Singapore, from 2008 to 2010. Most of the patients were self-referred, followed by those referred from private clinics and polyclinics. The diagnostic workup of suspected food-related reactions is shown in Fig. 1. Our centre uses published diagnostic decision thresholds for serum IgE tests and SPTs that can help predict the likelihood of an allergic reaction if a particular food is ingested. ${ }^{(6,7)}$ The decision to perform the challenge was based on these thresholds and the patient's clinical history. Patients generally did not proceed with an OFC if the treating physician felt that there was a high likelihood of a positive challenge, such as in the context of a recent life-threatening reaction to that food. Some patients underwent challenges for more than one food and a few patients were challenged repeatedly for the same food if their reactions were deemed to be subjective. 


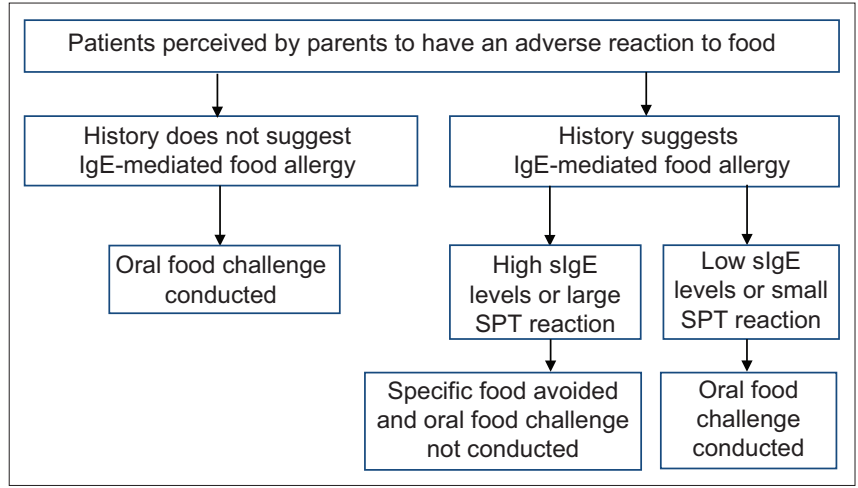

Fig. 1 Flowchart shows the diagnostic algorithm for the oral food challenge. IgE: immunoglobulin E; slgE: food-specific immunoglobulin E; SPT: skin prick test

The OFCs were performed at least 4-6 weeks after a previous food reaction and as open, unblinded challenges, according to protocol. ${ }^{\left({ }^{(8)}\right.}$ The patient was strictly observed for localised and/or systemic symptoms and signs. A challenge was deemed negative if the individual was able to tolerate an age-appropriate, mealsized portion of the suspected food ${ }^{(9)}$ without any untoward effects. During the procedure, medical personnel competent in resuscitation and resuscitation equipment were on standby. The patient was observed for a minimum of four hours after the OFC for any immediate-onset adverse reactions. A follow-up phone call was made the next day to all patients to track late adverse reactions.

\section{RESULTS}

A total of 197 OFCs were performed among 58 patients during the study period. The median age of the patients was six years and most of the patients were male (58.6\%). The patients were highly atopic, with $24.1 \%$ having concomitant asthma, $20.7 \%$ having allergic rhinitis and $39.7 \%$ having eczema. The characteristics of the study population and their indications for OFC are shown in Table I.

Tree nuts, peanuts, shellfish, egg and milk accounted for $84.8 \%$ of the OFCs (Table II). In the categories of perceived allergies by the parent, isolated positive allergy tests and possible aggravation of eczema, tree nuts were the most commonly avoided food (i.e. $68.2 \%, 62.7 \%$ and $31.3 \%$, respectively). Food sensitisation (i.e. either positive SPT or raised allergen-specific IgE levels) was detected in 51 patients, but only 3 (5.9\%) had a positive challenge. A total of 29 OFCs were performed on the patients who had a previous adverse reaction to the suspected food, but only four were positive. Interestingly, these four positive challenges yielded only mildly adverse reactions (typically cutaneous reactions).

Overall, 187 of the 197 (94.9\%) OFCs conducted were negative, with a particularly high negative challenge outcome in those patients with either isolated sensitisation or pure avoidance in the absence of a clinical reaction $(94.1 \%$ and $98.8 \%$, respectively). Table III lists the characteristics of the ten patients who had positive challenges. Of the ten positive challenges, five were to cow's milk, two were to peanuts, two were to treenuts and one was to shrimp. All the patients who had positive challenges had either a positive SPT or elevated allergen-specific
Table I. Characteristics of the patients who underwent oral food challenges during the study period $(n=58)$ and their indications.

\begin{tabular}{lc}
\hline Characteristic & No. (\%) \\
\hline Ethnicity & $27(46.6)$ \\
Chinese & $1(1.7)$ \\
Malay & $7(12.1)$ \\
Indian & $23(39.7)$ \\
Others & \\
Gender & $34(58.6)$ \\
Male & $24(41.4)$ \\
Female & $14(24.1)$ \\
Comorbidity & $12(20.7)$ \\
Asthma & $23(39.7)$ \\
Allergic rhinitis & \\
Eczema & $85(43.1)$ \\
Indication for challenge (n = 197) & \\
Foods avoided and never eaten due to parent's & $51(25.9)$ \\
perceived allergy in their child & $32(16.2)$ \\
Foods avoided due to previous positive allergy tests \\
Foods avoided due to possible worsening of eczema \\
Foods avoided due to previous reaction & $29.7)$ \\
\hline
\end{tabular}

IgE levels; seven of the ten patients $(70.0 \%)$ were female. Skin reactions were most common and occurred in eight of the ten patients who had positive challenges. None of the ten patients had gastrointestinal or cardiovascular symptoms. Reactions during the positive challenges did not result in death or hospitalisation, and resuscitation was not needed. All ten patients who had positive challenges were treated with antihistamines. None of the patients required epinephrine treatment.

\section{DISCUSSION}

In the present study, the evaluated indications for and outcomes of OFCs revealed that the majority of the children in the study had never eaten certain foods due to perceived parental fear that the foods may be allergenic. These perceptions are based on the possibility of cross-reactivity between foods and/or a family history of an adverse reaction. The introduction of a food that has been deliberately omitted from a child's diet may evoke feelings of fear and anxiety in both the child and parent. These feelings are real even though the perception that the child's symptoms are related to the food is usually subjective and may, in part, be augmented by a heightened public awareness and lack of knowledge of food allergies. To overcome such concerns, patients can be encouraged to undergo an OFC, which is an objective measure for detecting food allergies, in a controlled setting.

Negative OFCs abolish needless dietary restrictions and alleviate unnecessary patient and parental anxieties. ${ }^{(10)}$ In the present study, they resulted in the reintroduction of foods into diets and removal of food allergy labels. The largest number of OFCs was for tree nuts $(n=107)$ and most of the children involved had peanut allergies $(n=63)$. Nuts are commonly implicated, as they are a well-recognised cause of fatal foodinduced anaphylaxis, and have a history of contamination and cross-reactivity. However, studies have shown that blanket nut 
Table II. Frequency of oral food challenges (OFCs) conducted based on food types and according to the indications for OFC.

\begin{tabular}{|c|c|c|c|c|c|c|c|c|}
\hline \multirow[t]{2}{*}{ Indication for OFC } & \multicolumn{8}{|c|}{ OFCs based on food types [No. (\%)] } \\
\hline & Cow's milk & Egg & Tree nuts & Peanuts & Shellfish & Fish & Soy & Others \\
\hline $\begin{array}{l}\text { Foods avoided and never eaten due } \\
\text { to parent's perceived allergy }(n=85)\end{array}$ & 0 & $1(1.2)$ & $58(68.2)$ & $8(9.4)$ & $2(2.4)$ & $4(4.7)$ & $4(4.7)$ & $8(9.4)$ \\
\hline $\begin{array}{l}\text { Foods avoided due to previous } \\
\text { positive allergy tests }(n=51)\end{array}$ & $1(2.0)$ & $2(3.9)$ & $32(62.7)$ & $5(9.8)$ & $10(19.6)$ & 0 & 0 & $1(2.0)$ \\
\hline $\begin{array}{l}\text { Foods avoided due to possible } \\
\text { worsening of eczema }(n=32)\end{array}$ & $1(3.1)$ & $3(9.4)$ & $10(31.3)$ & $2(6.3)$ & $5(15.6)$ & $1(3.1)$ & $3(9.4)$ & $7(21.9)$ \\
\hline $\begin{array}{l}\text { Foods avoided due to previous } \\
\text { reaction }(n=29)\end{array}$ & $9(31.0)$ & $7(24.1)$ & $7(24.1)$ & $4(13.8)$ & 0 & 0 & 0 & $2(6.9)$ \\
\hline
\end{tabular}

Table III. Characteristics of patients with positive food challenges.

\begin{tabular}{|c|c|c|c|c|c|c|c|c|}
\hline $\begin{array}{l}\text { Age } \\
\text { (yr) }\end{array}$ & Gender & Ethnicity & Comorbidity & $\begin{array}{l}\text { Allergenic } \\
\text { food }\end{array}$ & $\begin{array}{c}\text { SPT } \\
(\mathrm{mm})\end{array}$ & $\begin{array}{c}\text { slgE } \\
(k U / L)\end{array}$ & Reaction & $\begin{array}{l}\text { Rescue } \\
\text { medicine }\end{array}$ \\
\hline 7 & Female & Others & Asthma, allergic rhinitis & Almond & - & 10.0 & Urticaria & Antihistamine \\
\hline 13 & Male & Chinese & Allergic rhinitis, eczema & Cow's milk & 7 & - & Urticaria, throat tightness & Antihistamine \\
\hline 6 & Male & Chinese & Eczema & Cow's milk & 9 & - & Urticaria & Antihistamine \\
\hline 8 & Female & Chinese & Eczema & Shrimp & - & 5.03 & Swelling of lips & Antihistamine \\
\hline 10 & Female & Others & Asthma & Peanut & 3 & - & Urticaria & Antihistamine \\
\hline 13 & Female & Others & Asthma & Peanut & 5 & - & Urticaria & Antihistamine \\
\hline 2 & Female & Chinese & None & Cow's milk & 3 & - & Urticaria & Antihistamine \\
\hline 4 & Female & Indian & Eczema & Cow's milk & 4 & - & Vomiting, urticaria & Antihistamine \\
\hline 6 & Male & Others & Asthma & Cow's milk & 10 & - & Vomiting & Antihistamine \\
\hline 4 & Female & Others & Allergic rhinitis, eczema & Hazelnut & - & 2.84 & Urticaria & Antihistamine \\
\hline
\end{tabular}

Others: other races; slgE: food-specific immunoglobulin E; SPT: skin prick test

avoidance is not advisable due to the negative impact on lifestyle and nutrition. ${ }^{(11)}$ In addition, it has been suggested that the introduction of a single tree nut may attenuate hypersensitivity to other tree nuts. ${ }^{(12)}$ In other words, it would be useful to introduce nuts for which the child tests negative into the child's diet. In cases where there is parental or patient fear, OFCs should be encouraged. A controlled setting, such as the one provided by our centre, gives highly anxious patients and their parents objective evidence of their allergy status. ${ }^{(13)}$ After the OFC, physicians should continue providing support to these patients and their parents, to help them to reintroduce the foods into their diets. ${ }^{(14}$

In the present study, 187 of the 197 (94.9\%) OFCs performed were negative and safe (162 of these 197 OFCs were conducted in an outpatient setting). The incidence of negative OFCs is consistent with that published in other reports, ${ }^{(13,15)}$ suggesting that OFCs in carefully selected populations are safe and practical, unlike the traditional double-blind, placebo-controlled challenges that are more time-consuming and less cost-effective. However, physicians should ensure that the safety of the patient is not compromised in the quest for aggressive challenges. Patient selection for OFCs is not always straightforward. The rate of developing tolerance varies in patients who have a clear history of a previous reaction. In patients without prior reactions but who are sensitised, positive predictive values in tests are not always reliable. In the present study, we had a positive reaction rate of $5.1 \%$, which was considerably lower than that reported in other studies. ${ }^{(16)}$ This may be attributed to the fact that avoidance due to fear rather than true allergy was the diagnosis in the majority of our patients; that is, these children may not have been allergic to the abstained foods in the first place. These children can be classified as a low-risk cohort whose pretest probability of clinical reactivity to foods is low. However, it is in this exact population that the risk-to-benefit ratio is optimal for performing an OFC, a finding that is corroborated by other studies. ${ }^{(13)}$

None of the patients in the present study experienced lifethreatening reactions and no epinephrine was dispensed. This may in part be due to patient selection. None of the ten patients who had a positive challenge had a negative SPT or negative allergen-specific IgE test. It is not surprising that a positive challenge was more frequently identified among the patients who had a previous positive reaction (13.7\%). Conversely, the likelihood of a negative challenge is high if there was only sensitisation or pure avoidance in the absence of a clinical reaction (94.1\% and $98.8 \%$, respectively). As other studies have demonstrated, reliance on perception, subjective symptoms and evidence of sensitisation is a poor predictor of true food allergies, in the absence of clinical reactivity. ${ }^{(5)}$ This is especially true for patients with atopic dermatitis, many of whom mistakenly avoid foods that they believe are causing eczema flare-ups, but neglect vital, basic skin care. ${ }^{(17)}$

The present study had its limitations. As it was a retrospective study, the accuracy of the clinical history, the choice of test performed (SPT or allergen-specific IgE test), and the decision to administer an OFC depended on the attending physician. However, as our centre followed a standard protocol for treating food allergies, it is likely that practices were largely consistent 
and were unlikely to have significantly affected the study's results. Furthermore, some of the patients may have developed tolerance while awaiting their OFC. The OFCs were also unblinded and the final dose was not standardised according to protein content; instead, doses were determined by the treating clinician together with the parents based on the usual meal-sized portion of food that the child would eat. This was done so that the test conditions reflected the likely situation in which the child would continue to eat the food following a negative challenge. Given the high rate of negative challenges, the lack of a double-blind, placebocontrolled food challenge is unlikely to have significantly influenced the reported outcomes of the OFCs. As all positive challenges had objective evidence of an IgE-mediated allergic reaction or a positive SPT, we believe that the lack of blinding in the present study was not the cause of the positive challenges.

To conclude, the use of OFCs has revealed a discrepancy between what parents perceive and objective diagnoses of adverse reactions to food in their children. OFCs are an objective measure of true food allergies and are safe when performed in a supervised setting. Following negative challenges, food introduction was achieved in the majority of the patients, unnecessary food labels were removed and families were empowered to make better food choices. The OFCs were performed as open challenges and were safe, as the patients were carefully selected based on detailed history, SPT results and allergen-specific IgE levels.

\section{REFERENCES}

1. Rona RJ, Keil T, Summers C, et al. The prevalence of food allergy: a metaanalysis. J Allergy Clin Immunol 2007; 120:638-46.

2. Eggesbø M, Halvorsen R, Tambs K, Botten G. Prevalence of parentally perceived adverse reactions to food in young children. Pediatr Allergy Immunol 1999; 10:122-32.
3. Clark AT, Ewan PW. Interpretation of tests for nut allergy in one thousand patients, in relation to allergy or tolerance. Clin Exp Allergy 2003; 33:1041-5.

4. Eigenmann PA, Sicherer SH, Borkowski TA, Cohen BA, Sampson HA. Prevalence of IgE-mediated food allergy among children with atopic dermatitis. Pediatrics 1998; 101:E8.

5. Fleischer DM, Bock SA, Spears GC, et al. Oral food challenges in children with a diagnosis of food allergy. J Pediatr 2011; 158:578-583.e1.

6. Sampson HA. Utility of food-specific IgE concentrations in predicting symptomatic food allergy. J Allergy Clin Immunol 2001; 107:891-6.

7. Hill DJ, Heine RG, Hosking CS. The diagnostic value of skin prick testing in children with food allergy. Pediatr Allergy Immunol 2004; 15:435-41.

8. Bindslev-Jensen C, Ballmer-Weber BK, Bengtsson $U$, et al; European Academy of Allergology and Clinical Immunology. Standardization of food challenges in patients with immediate reactions to foods--position paper from the European Academy of Allergology and Clinical Immunology. Allergy 2004; 59:690-7.

9. Nowak-Wegrzyn, A, Assa'ad AH, Bahna SL, et al; Adverse Reactions to Food Committee of American Academy of Allergy, Asthma \& Immunology. Work Group report: oral food challenge testing. J Allergy Clin Immunol 2009; $123(6$ Suppl):S365-83

10. Sivaraj H, Rajakulendran M, Lee BW, Shek LP. Challenges faced by expatriate children with food allergy in an Asian country. Ann Allergy Asthma Immunol 2010; 105:323-4.

11. Ball H, Luyt D, Bravin K, Kirk K. Single nut or total nut avoidance in nut allergic children: outcome of nut challenges to guide exclusion diets. Pediatr Allergy Immunol 2011; 22:808-12.

12. Kulis M, Li Y, Lane H, Pons L, Burks W. Single-tree nut immunotherapy attenuates allergic reactions in mice with hypersensitivity to multiple tree nuts. J Allergy Clin Immunol 2011; 127:81-8.

13. Lieberman JA, Cox AL, Vitale M, Sampson HA. Outcomes of office-based, open food challenges in the management of food allergy. J Allergy Clin Immunol 2011; 128:1120-2.

14. Strinnholm A, Brulin C, Lindh V. Experiences of double-blind, placebocontrolled food challenges (DBPCFC): a qualitative analysis of mothers' experiences. J Child Health Care 2010; 14:179-88.

15. Mankad VS, Williams LW, Lee LA, et al. Safety of open food challenges in the office setting. Ann Allergy Asthma Immunol 2008; 100:469-74.

16. Perry TT, Matsui EC, Conover-Walker MK, Wood RA. Risk of oral food challenges. J Allergy Clin Immunol 2004; 114:1164-8.

17. Rancé F. Food allergy in children suffering from atopic eczema. Pediatr Allergy Immunol 2008; 19:279-84; quiz 285. 